\title{
Atom Probe Tomography Using a Wavelength-Tunable Femtosecond-Pulsed Coherent Extreme Ultraviolet Light Source
}

\author{
Ann N. Chiaramonti ${ }^{*}$, Luis Miaja-Avila ${ }^{1}$, Paul T. Blanchard ${ }^{1}$, David R. Diercks², Brian P. Gorman ${ }^{2}$, \\ and Norman A. Sanford ${ }^{1}$ \\ 1. National Institute of Standards and Technology, Boulder, CO, USA \\ 2. Metallurgical and Materials Engineering, Colorado School of Mines, Golden, CO, USA \\ * Corresponding author: chiaramonti@nist.gov
}

Laser-pulsed atom probe tomography (LAPT) is a powerful tool for materials characterization due to its desirable combination of high spatial resolution and analytical sensitivity. In state-of-the-art LAPT, the thermal pulse resulting from a near-ultraviolet (NUV) laser ( $\mathrm{E} \approx 3.5 \mathrm{eV}$ to $3.6 \mathrm{eV} ; \lambda \approx 355 \mathrm{~nm}$ to $343 \mathrm{~nm}$ ) incident on the sample provides the energy to overcome the activation barrier for field ion evaporation. LAPT has been used successfully to characterize a wide range of materials including metals, semiconductors, insulators, biological materials, and even liquids. However, the thermal process is not without drawbacks. LAPT data quality can be degraded due to for example: thermal tails that limit sensitivity; the formation of cluster ions that may have isobaric overlap with elemental species; undetected neutral species which can adversely influence composition measurements; and unresolvable multiple hits which result in a loss of information. Data loss due to multiple hits and neutral species is particularly problematic for many ionic and covalent materials; it can limit the recovery of bulk stoichiometry or composition to a narrow range of experimental conditions, if at all [1,2].

Ionizing radiation in the extreme ultraviolet (EUV) region of the electromagnetic spectrum $(\approx 10 \mathrm{eV}$ to $\approx 100 \mathrm{eV} ; 124 \mathrm{~nm}$ to $12 \mathrm{~nm}$ ) offers potential new athermal field ionization pathways (direct photoionization and Auger decay) for atom probe tomography. Much of the EUV photon energy band is above the work function and ionization potential of any naturally occurring element, and photoionization crosssections peak in the EUV band across the entire periodic table [3]. EUV is highly absorbed within only the first few nm of the sample surface and may also offer a potential in situ method for imaging the specimen shape using coherent diffractive imaging [4].

Instrument design (Figure 1) and initial results from the world's first EUV radiation-pulsed atom probe tomograph are presented. This instrument uses tunable wavelength (photon energy) femtosecond-pulsed coherent EUV radiation from phase-matched high harmonic generation in a hollow waveguide. Initial experiments demonstrate EUV $(41.85 \mathrm{eV} ; \lambda=29.6 \mathrm{~nm})$ radiation-pulsed field ion emission in $\mathrm{SiO}_{2}, \mathrm{BaTiO}_{3}$, $\mathrm{GaN}, \mathrm{GaN}: \mathrm{Mg}, \mathrm{GaN} / \mathrm{InGaN}, \mathrm{Si}$, and Al. Time-independent background levels, delayed evaporation tails, peak widths, charge state ratios, multiple hit counts, and the relative number of cluster ions will be compared to NUV LAPT $(E \approx 3.5 \mathrm{eV} ; \lambda=355 \mathrm{~nm}$ ) experiments on the same samples and specimens (Figure 2) [5].

\section{References:}

[1] Mancini, L. et al., J. Phys. Chem. C 118 (2014), p. 24136.

[2] Diercks, D.R. et al., J. Appl. Phys. 114 (2013), p. 184903.

[3] Yeh, J.-J. and I. Landau, At. Data Nucl. Data Tables 32 (1985), p. 1.

[4] Gardner, D.F. et al., Nature Photonics 11 (2017), p. 259.

[5] This project was made possible with material support from CAMECA Instruments through a Cooperative Research and Development Agreement. Commercial equipment is identified in this paper in order to specify the experimental procedure adequately. Such identification is not intended to imply recommendation or 
endorsement by the National Institute of Standards and Technology, nor is it intended to imply that the materials or equipment identified are necessarily the best available for the purpose.

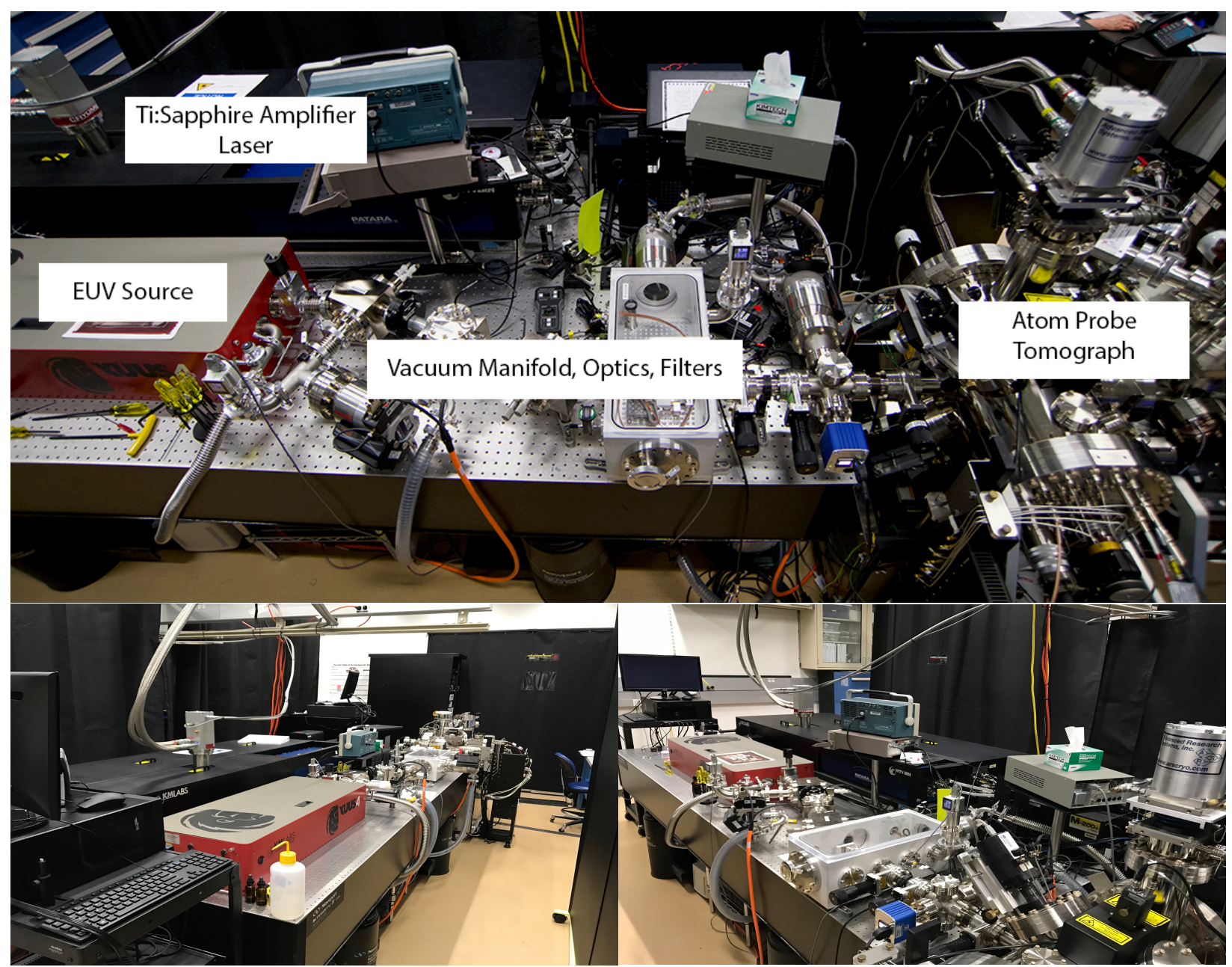

Figure 1: Extreme ultraviolet atom probe tomograph at NIST Boulder Laboratories. (Top) Top view. (Bottom Left) Looking toward the atom probe. (Bottom Right) Looking toward the EUV source.
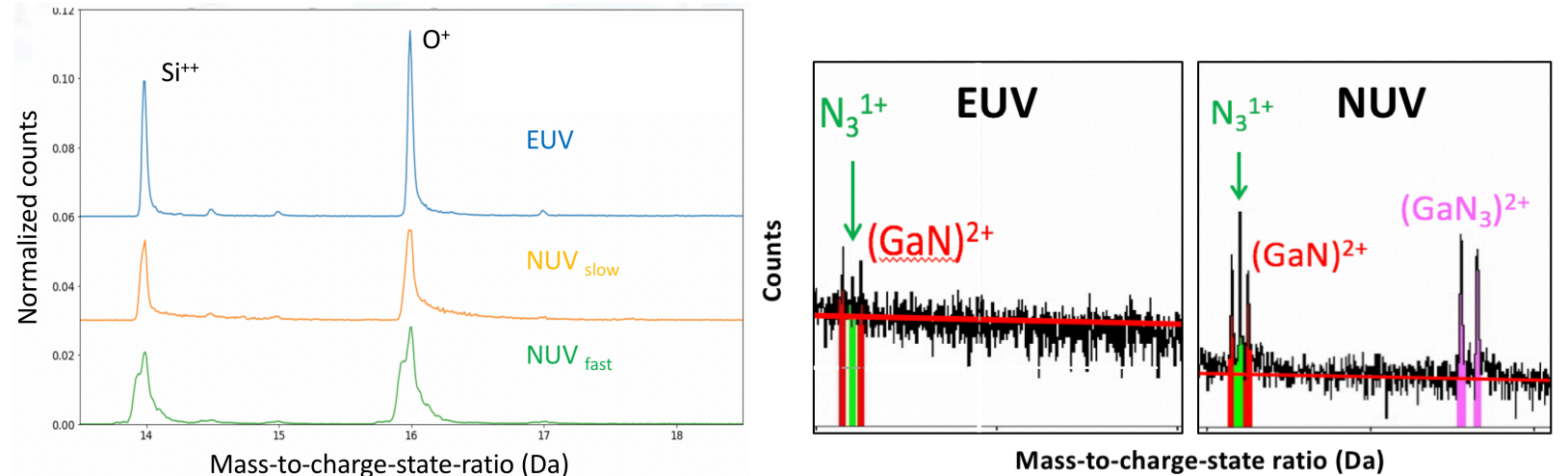

Figure 2: (Left) Normalized, background subtracted portion of the mass spectrum of $\mathrm{SiO}_{2}$ comparing EUV $(10 \mathrm{kHz}), \mathrm{NUV}(25 \mathrm{kHz})$, and NUV $(500 \mathrm{kHz})$. (Right) Raw mass spectrum of GaN demonstrating elimination of the $\mathrm{GaN}_{3}{ }^{++}$cluster and a decrease in the relative intensity of the $\mathrm{N}_{3}{ }^{+}$and $\mathrm{GaN}^{++}$clusters in the EUV data compared to NUV data. An estimate of the time-independent background is in red. 